Acta Physica Academiae Scientiarum Hungaricae, Tomus 24 (4), pp. $391-402$ (1968)

\title{
ON THE EFFECT OF THE INCLUSION OF OVERLAP IN TIGHT-BINDING BAND CALCULATIONS OF SOLIDS
}

\author{
By
}

\author{
G. Del RE*, J. Ladik
}

CENTRAL RESEARCH INSTITUTE FOR CHEMISTRY OF THE HUNGARIAN ACADEMY OF SCIENCES, BUDAPEST and

M. Carpentieri

INSTITUTE OF THEORETICAL PHYSICS, UNIVERSITY OF NAPLES, NAPLES, ITALY

(Presented by G. Schay. - Received 10. VIII. 1967)

To investigate the effect of the inclusion of overlap integrals in the tight binding approximation the energy band structure of a model polymer containing two centres and four orbitals in the elementary cells has been calculated. In the first series of calculations the value of the intracell overlap integral, in the second and third series the large, respectively small intercell overlap integrals have been systematically changed. According to the results obtained the changes of both the intracell and intercell overlap integrals (also in the case of the small intercell ones) cause rather large changes in the band structures. Therefore, the usual neglect of overlap integrals in the tight binding approximation of the band structure of polymers is not justified.

\section{Introduction}

Tight binding calculations on crystals may be considered as an extension to infinite periodic systems of the well-known LCAO-MO calculations for molecules. So far, examples of application of such calculations to solids have been given in the semiempirical approximations neglecting overlap, like the Hückel method and the so-called P-P.P method $[1,2]$. Now, in the case of molecules, neglect of overlap, although it can usually be compensated through a careful choice of the parameters, may lead to results quite different from those that would be obtained if overlap were included. For instance, if the relationship [3]

$$
S_{\mu \nu}=\lambda H_{\mu \nu}
$$

with $\lambda$ a general constant, holds between the overlap integrals $S_{\mu, v}$ and the off-diagonal elements $H_{\mu, v}$ of the Hamiltonian matrix (referring to the pair of atoms $\mu, v)$, the eigenvalues $\varepsilon_{i}$ defined by the equation

$$
\mathbf{H C}=\mathbf{S C} \epsilon
$$

are related to those of a suitably "orthogonalized" equation

$$
\mathbf{H}^{\prime} \mathbf{C}=\mathbf{C}^{\prime} \epsilon^{\prime}
$$

* Permanent address: Roma, Gruppo Chimica Teorica CNR. Via Ce so 7, 00161 Roma 
through the equation [4]:

$$
\varepsilon_{i}=\frac{\varepsilon_{i}^{\prime}}{1+\lambda \varepsilon_{i}^{\prime}}
$$

This shows that the numerical values of the $\varepsilon_{i}^{\prime \prime}$ 's are not only different from those of the $\varepsilon_{i}$ 's but also their spacing is completely different: if $\varepsilon_{i}^{\prime}$ and $\varepsilon_{j}^{\prime}$ are symmetric with respect to the zero level, $\varepsilon_{i}$ and $\varepsilon_{j}$ will not behave so: the higher level will be pushed further from the zero-level, whereas the lower will become closer to the zero-level.

These considerations apply qualitatively in most cases, even if a general algebraic proof is not easy to give. In particular, they apply when $\mathbf{H}^{\prime}=\mathbf{H}$ and the only difference between (2) and (3) is that the off-diagonal elements of the overlap matrix are neglected. On the other hand, they do not apply, of course, if Eq. (3) is adopted under the assumption that the "atomic" orbital basis is a strietly orthogonal one (as is often obtained by LöwDIN's [5] symmetric orthogonalization). However, an orthogonalization procedure is difficult to apply to infinite lattices, and, moreover, it would give a fully non-localised basis, thus leading to difficulties of interpretation particularly serious for the treatment of local perturbations.

A general formalism explicitly including overlap in a tight-binding SCF treatment of one-, two- and three-dimensional crystals has been developed [6]. However, as has been mentioned, a direct algebraic analysis of the importance of overlap in the corresponding calculations is very difficult, if not impossible. On the other hand, calculations neglecting overlap are usually the most straightforward ones in the empirical versions of the LCAO crystal orbital method. Therefore, a numerical analysis of the effect of overlap on such calculations in desirable. This is what the present paper is devoted to. In order to make the interpretation of the results easier, we shall consider a simple linear chain, with two atoms in the unit cell. The effect on the bands of changing the overlap integrals within the unit cell, and between adjacent unit cells, when the Hamiltonian matrix is kept constant, will be the subject of our study.

\section{Description of the calculations}

To calculate the energy bands of a solid in the tight-binding (LCAO) approximation, with inclusion of overlap, one has to solve the matrix equation [6]

$$
\mathbf{H}(k) \mathbf{C}(k)=\mathbf{S}(k) \mathbf{C}(k) \epsilon(k)
$$

which is the counterpart of $\mathrm{Eq}$. (2) in the case of a crystal. There, $k$ is the wave vector which specifies the levels in the $n$ bands one encounters in this type of calculation, and $\mathbf{H}(k)$ and $\mathbf{S}(k)$ are, respectively $n \times n$ complex hermitian 
Hamiltonian and overlap matrices, $n$ being the number of orbitals of the unit cell. The elements of the real diagonal matrix $\epsilon(k)$ are the corresponding energy levels in the different bands; matrix $\mathrm{C}(k)$ is the eigenvector matrix, obeying the normalisation condition

$$
\mathbf{C}^{+}(k) \mathbf{S}(k) \mathbf{C}(k)=\mathbf{I}
$$

As is well known [5], one can solve Eq. (5) most easily by transforming it into the new equation:

$$
\mathbf{F}(k) \mathbf{B}(k)=\mathbf{B}(k) \in(k)
$$

where*

$$
\mathbf{F}(k)=\mathbf{S}(k)^{-1 / 2} \mathbf{H S}(k)^{-1 / 2}
$$

and

$$
\mathbf{B}(k)=\mathbf{S}(k)^{1 / 2} \mathbf{C}(k)
$$

The square root and inverse square root of $S(k)$ are easily calculated by finding the diagonal form $s(k)$ of $S(k)$ from the eigenvalue equation

$$
\mathbf{S}(k) \mathbf{V}(k)=\mathbf{V}(k) \mathbf{s}(k)
$$

After having diagonalized the matrix $\mathbf{S}(k)$, one forms the diagonal matrices $\mathbf{s}(k)^{12}, s(k)^{-1 / 2}$, respectively, and uses the equations

$$
\mathbf{S}(k)^{+1 / 2}=\mathbf{V}(k) \mathbf{s}(k)^{+1 / 2} \mathbf{V}^{+}(k) \text { and } \mathbf{S}(k)^{-1 / 2}=\mathbf{V}(k) s(k)^{-1 / 2} \mathbf{V}^{+}(k)
$$

The eigenvalue prohlem (9) can be solved by rewriting it in a real form

$$
\mathbf{S}^{\prime}(k)=\left(\begin{array}{rr}
\operatorname{Re}[\mathbf{S}(k)] & -\operatorname{Im}[\mathbf{S}(k)] \\
\operatorname{Im}[\mathbf{S}(k)] & \operatorname{Re}[\mathbf{S}(k)]
\end{array}\right)\left(\begin{array}{l}
\mathbf{V}_{1}(k) \\
\mathbf{V}_{2}(k)
\end{array}\right)=\left(\begin{array}{l}
\mathbf{V}_{1}(k) \\
\mathbf{V}_{2}(k)
\end{array}\right) \mathbf{s}^{\prime}(k)
$$

where

$$
\mathbf{V}_{1}(k)=\operatorname{Re}[\mathbf{V}(k)], \mathbf{V}_{2}(k)=\operatorname{Im}[\mathbf{V}(k)]
$$

In the diagonal matrix $s^{\prime}(k)$ each eigenvalue of $\mathbf{S}(k)$ occurs twice. In a similar way we can rewrite $(6)$ as

$$
\begin{gathered}
\mathbf{F}(k)=\left(\begin{array}{rr}
\operatorname{Re}[\mathbf{F}(k)] & -\operatorname{Im}[\mathbf{F}(k)] \\
\operatorname{Im}[\mathbf{F}(k)] & \operatorname{Re}[\mathbf{F}(k)]
\end{array}\right)\left(\begin{array}{l}
\mathbf{B}_{1}(k) \\
\mathbf{B}_{2}(k)
\end{array}\right)=\left(\begin{array}{l}
\mathbf{B}_{1}(k) \\
\mathbf{B}_{2}(k)
\end{array}\right) \mathbf{\epsilon}^{\prime}(k) \\
\left.\left(\mathbf{B}_{1}(k)\right]=\operatorname{Re}[\mathbf{B}(k)] \quad \text { and } \quad \mathbf{B}_{2}(k)=\operatorname{Im}[\mathbf{B}(k)]\right) .
\end{gathered}
$$

\footnotetext{
* A slightly different but entirely equivalent form of Eqs. (7) and (9) was given in [6].
} 
Taking into account Eq. (7), it can be shown that its real and imaginary parts are

$$
\begin{aligned}
\operatorname{Re}[\mathbf{F}(k)] & =\operatorname{Re}[\mathbf{C}(k)][\operatorname{Re}[\mathbf{H}(k)] \operatorname{Re}[\mathbf{C}(k)]-\operatorname{Im}[\mathbf{H}(k)] \operatorname{Im}[\mathbf{C}(k)]]- \\
& -\operatorname{Im}[\mathbf{C}(k)][\operatorname{Im}[\mathbf{H}(k)] \operatorname{Re}[\mathbf{C}(k)]+\operatorname{Re}[\mathbf{H}(k)] \operatorname{Im}[\mathbf{C}(k)]] \\
\operatorname{Im}[\mathbf{F}(k)] & =\operatorname{Re}[\mathbf{C}(k)][\operatorname{Re}[\mathbf{H}(k)] \operatorname{Im}[\mathbf{C}(k)]+\operatorname{Im}[\mathbf{H}(k)] \operatorname{Re}[\mathbf{C}(k)]]+ \\
& +\operatorname{Im}[\mathbf{C}(k)][\operatorname{Re}[\mathbf{H}(k)] \operatorname{Re}[\mathbf{C}(k)]-\operatorname{Im}[\mathbf{H}(k)] \operatorname{Im}[\mathbf{C}(k)]]
\end{aligned}
$$

respectively. Here the matrices $\operatorname{Re}[\mathrm{C}(\mathrm{k})]$ and $\operatorname{Im}[\mathrm{C}(\mathrm{k})]$ are defined as

$$
\begin{aligned}
& \operatorname{Re}[\mathbf{C}(k)]=\mathbf{V}_{1}(k) s(k)^{-1 / 2} \mathbf{V}_{1}^{+}(k)+\mathbf{V}_{2}(k) s(k)^{-1 / 2} \mathbf{V}_{2}(k)^{+} \\
& \operatorname{Im}[\mathbf{C}(k)]=-\mathbf{V}_{1}(k) s(k)^{-1 / 2} \mathbf{V}_{2}(k)+\mathbf{V}_{2}(k) s(k)^{-1 / 2} \mathbf{V}_{1}(k)
\end{aligned}
$$

Equations (10) and (11) are those actually used for practical calculations. When $\mathbf{S}(k)$ is the identity matrix, $\mathbf{B}(k)$ is identical with $\mathbf{C}(k), \mathbf{F}(k)$ coincides with $\mathbf{H}(k)$ and, for the case of a linear chain, it gives results exemplified in previous calculations on rather complicated polymers by one of us [7]. However, as has been said, to study the effect of increasing off-diagonal elements of $\mathbf{S}(k)$ on the energy matrix $\epsilon(k)$ and hence on the shapes and distributions of the bands, it is useful to consider a model system, namely the linear chain of Fig. 1. Here the lone pairs of the (fictitious) $X$ atoms are assumed to be completely localized, so that only three (unspecified) orbitals are considered, and the atoms $Y$ are assumed to share only one orbital with the bond system (in practice, the chain of Fig. 1 could be one of, say, $\mathrm{N}-\mathrm{H}$ groups).

As a further simplification of our model calculations we shall work on the model system shown in Fig. 1 in the nearest neighbour approximation. Here the matrices $\mathbf{S}(k)$ and $\mathbf{H}(k)$ * will have the simple form [6]

$$
\mathbf{S}(k)=\mathbf{1}+\Delta+\mathbf{S}^{+} e^{i k}+\mathbf{S}^{-} e^{-i k}
$$

and

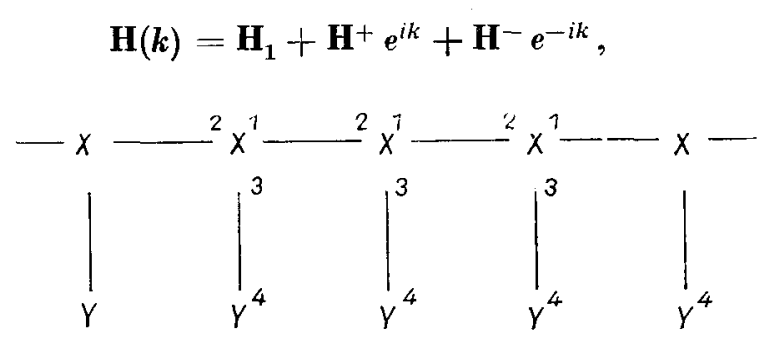

Fig. 1. The model system used in the calculation

* In the case of a linear chain instead of a vector $k$ we have a scalar wave number $k$. 
respectively. Here 1 is the unit matrix, $\Delta$ is the matrix of the overlap integrals between orbitals belonging to the same elementary cell and the elements of the matrices $\mathbf{S}^{+}$and $\mathbf{S}^{-}$, respectively, are given by the overlap integrals between the orbitals of an elementary cell and its right and left neighbours, respectively. The matrices $\mathbf{H}_{1}, \mathbf{H}^{+}$and $\mathbf{H}^{-}$, respectively, are defined in a similar way. From $(14 a)$ and $(14 b)$ we obtain

$$
\begin{aligned}
\operatorname{Re}[\mathbf{S}(k)] & =\mathbf{I}+\Delta+\left(\mathbf{S}^{+}+\mathbf{S}^{-}\right) \cos k, \\
\operatorname{Im}[\mathbf{S}(k)] & =\left(\mathbf{S}^{+}-\mathbf{S}^{-}\right) \sin k, \\
\operatorname{Re}[\mathbf{H}(k)] & =\mathbf{H}_{1}+\left(\mathbf{H}^{+}+\mathbf{H}^{-}\right) \cos k, \\
\operatorname{Im}[\mathbf{H}(k)] & =\left(\mathbf{H}^{+}-\mathbf{H}^{-}\right) \sin k .
\end{aligned}
$$

As we have only four orbitals in each elementary cell our complex matrices will be of order of 4 (the real matrices of the order of 8). As input matrices we have given the matrices $\Delta, \mathbf{S}^{+}, \mathbf{H}_{1}$ and $\mathbf{H}^{+}\left(\right.$by $\operatorname{symmetry} \mathbf{S}^{-}(k)=$ $\left.=\left[\mathbf{S}^{+}(k)\right]^{t r}, \mathbf{H}^{-}(k)=\left[\mathbf{H}^{+}(k)\right]^{t r}\right)$.

Our analysis has been carried out with fixed $\mathrm{H}_{1}$ and $\mathrm{H}^{+}$matrices. In matrix $H_{1} \propto$ values corresponding to the 4 orbitals and the bond integral value corresponding $\mathrm{N}-\mathrm{H}$ bond $\left(\beta_{3,4}\right)$ were chosen different from zero. In the $\mathbf{H}^{+}$matrix we have taken non-zero values for the elements corresponding to those elements $S_{\mu, \nu}$ of the overlap matrix $\mathbf{S}$ which have been assumed to be different from zero (see below*). In the matrix $\Delta$ we have considered only the overlap integral corresponding to the $\mathrm{X}-\mathrm{Y}$ bond. In the $\mathrm{S}^{+}$matrix the overlap integrals $S_{1,2}^{+}$(the $X-X$ bond) and also the integrals $S_{1,3}^{+}, S_{1,4}^{+}, S_{3,2}^{+}, S_{3,3}^{+}, S_{3,4}^{+}$, $\mathrm{S}_{4,9}^{+}, \mathrm{S}_{4,3}^{+}, \mathrm{S}_{4,4}^{+}$have been taken into account.

In a first series of calculations we have taken for the only non-zero element of $A, \mathrm{~S}_{3,4}$ the values $0(0.20) 0.60,0.70,0.80$ and 0.90 , respectively. The matrices $\mathbf{S}^{+}, \mathbf{H}_{1}$ and $\mathbf{H}^{+}$were kept constant:

$$
\begin{gathered}
\mathbf{S}^{+}=\left(\begin{array}{cccc}
0 & 0,70 & 0,10 & 0,10 \\
0 & 0 & 0 & 0 \\
0 & 0,10 & 0,10 & 0,10 \\
0 & 0,10 & 0,10 & 0,10
\end{array}\right), \quad \mathbf{H}_{1}=\left(\begin{array}{cccc}
0,30 & 0 & 0 & 0 \\
0 & 0,30 & 0 & 0 \\
0 & 0 & 0,30 & 0,45 \\
0 & 0 & 0,45 & 0,20
\end{array}\right) \\
\mathbf{H}^{+}=\left(\begin{array}{cccc}
0 & 1,00 & 0,15 & 0,15 \\
0 & 0 & 0 & 0 \\
0 & 0,15 & 0,15 & 0,15 \\
0 & 0,15 & 0,15 & 0,15
\end{array}\right) .
\end{gathered}
$$

\footnotetext{
* The elements in the matrices $\mathbf{H}_{1}$ and $\mathbf{H}^{+}$(see below) are given in $\beta$ units. They have been chosen so as to correspond in order of magnitude with those used in Hückel-type calculations on $\sigma$ systems [8].
} 
The calculations were executed for the $k$ values

$$
0\left(\frac{\pi}{4}\right) \pi
$$

In a second series of calculations we have kept $S_{3,4}$ constant and we have chosen $S_{34}=0.70$. Now we have changed the integral $S_{1,2}^{+}$for which the values 0 (0.20) 0.80 were taken. All the other elements of $S^{+}$, as well as those of $\mathbf{H}_{1}$ and $\mathrm{H}^{+}$were the same as in the first series of calculations. These calculations have also been performed for the values

$$
k=0\left(\frac{\pi}{4}\right) \pi
$$

In a last series of calculations, we have taken $\Delta$ as in the second case, we have chosen for $S_{3,4}^{+}=0.70$ and we have simultaneously changed all the other non-zero elements of $S^{+}$choosing for them the values $0,0.05,0.10$ and 0.15 , respectively. The matrices $H_{1}$ and $H^{+}$were the same as in the first and second series of calculations.

\section{Results and discussion}

Tables I, II and III, and Figs. 2, 3 illustrate the band structures obtained for the three series of calculations.

In the Tables each column gives a band calculated by the parameter value indicated in the first rows of the Tables. In Table III $\mathrm{S}_{\mu \nu}^{+}$refers to all the simultaneously changed non-zero elements of the matrix $\mathbf{S}^{+}$. The two values in italic type in each column indicate the limits of the bands, which are always at $\mathbf{k}=0$ and $\mathbf{k}=\pi$.

We shall discuss first some general features and the effect of the change of the overlap integral relating to the intra-cell $X-Y$ bond on the position, width and shape of the four bands obtained in the calculations described in the previous Section. The considerations given in the Introduction suggest that upon introduction of overlap the bands originally symmetric with respect to the zero-point implicitly chosen in setting up the matrix $H$ would be displaced in an unsymmetrical way: the lower band would be shifted towards the zero level and become narrower; the upper band would be shifted upwards and become wider. This behaviour is also to be expected in a general qualitative sense for bands not symmetric with respect to the zero level. As is seen from the Tables and the Figures, this is more or less the case for our model polymer, but each overlap integral has effects which need to be discussed separately. 


\section{Table I}

The effect of the change of the intracell overlap integral on the band structure of the investigated model system

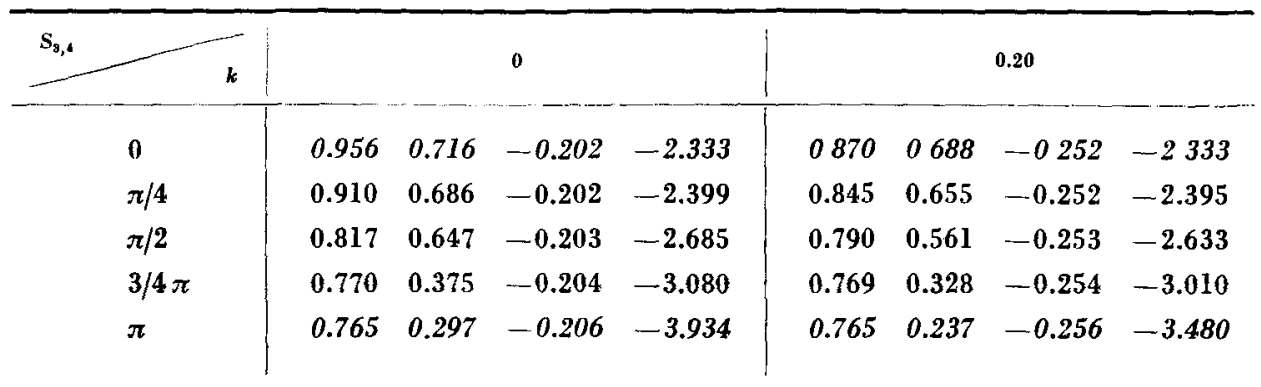

\begin{tabular}{l|llll|lllll}
\hline \multicolumn{2}{l|}{} & \multicolumn{5}{|c|}{0.40} & \multicolumn{4}{|c}{0.60} \\
\hline 0 & 0.824 & 0.645 & -0.336 & -2.333 & 0.803 & 0.596 & -0.503 & -2.333 \\
$\pi / 4$ & 0.810 & 0.604 & -0.336 & -2.391 & 0.793 & 0.558 & -0.503 & -2.387 \\
$\pi / 2$ & 0.781 & 0.490 & -0.337 & -2.595 & 0.776 & 0.434 & -0.504 & -2.566 \\
$3 / 4 \pi$ & 0.768 & 0.290 & -0.338 & -2.940 & 0.767 & 0.259 & -0.505 & -2.872 \\
$\pi$ & 0.765 & 0.197 & -0.339 & -3.229 & 0.765 & 0.170 & -0.506 & -3.070
\end{tabular}

\begin{tabular}{|c|c|c|c|c|c|c|c|c|}
\hline & \multicolumn{4}{|c|}{0.70} & \multicolumn{4}{|c|}{0.80} \\
\hline 0 & 0.796 & 0.572 & -0.670 & -2.333 & 0.792 & 0.550 & -1.004 & -2.333 \\
\hline$\pi / 4$ & 0.788 & 0.533 & -0.670 & -2.385 & 0.795 & 0.510 & -1.004 & -2.383 \\
\hline$\pi / 2$ & 0.775 & 0.410 & -0.761 & -2.553 & 0.774 & 0.389 & -1.004 & -2.542 \\
\hline $3 / 4 \pi$ & 0.767 & 0.243 & -0.672 & -2.834 & 0.767 & 0.229 & -1.005 & -2.801 \\
\hline$\pi$ & 0.765 & 0.159 & -0.673 & -3.010 & 0.765 & 0.155 & -1.005 & -2.950 \\
\hline
\end{tabular}

\begin{tabular}{l|llll}
\hline \multicolumn{1}{l|}{$s_{s, 4}$} & \multicolumn{4}{|c}{0.90} \\
\hline 0 & 0.788 & 0.529 & -2.004 & -2.333 \\
$\pi / 4$ & 0.782 & 0.490 & -2.004 & -2.381 \\
$\pi / 2$ & 0.773 & 0.370 & -2.004 & -2.533 \\
$3 / 4 \pi$ & 0.767 & 0.217 & -2.003 & -2.775 \\
$\pi$ & 0.765 & 0.141 & -2.003 & -2.918
\end{tabular}




\section{Table II}

The effect of the change of the larger intercell overlap integral on the band structure of the investigated model system

\begin{tabular}{|c|c|c|c|c|c|c|c|c|}
\hline $\begin{array}{ll} & s_{1,2}^{\perp}\end{array}$ & \multicolumn{4}{|c|}{0} & \multicolumn{4}{|c|}{0.20} \\
\hline 0 & 1.301 & 0.600 & -0.670 & -0.600 & 1.089 & 0.596 & -0.670 & -0.875 \\
\hline$\pi / 4$ & 1.301 & 0.550 & -0.669 & -0.640 & 1.088 & 0.547 & -0.670 & -0.910 \\
\hline$\pi / 2$ & 1.300 & 0.413 & -0.668 & -0.755 & 1.086 & 0.413 & -0.670 & -0.942 \\
\hline $3 / 4 \pi$ & 1.300 & 0.234 & -0.668 & -0.860 & 1.085 & 0.236 & -0.670 & -1.020 \\
\hline$\pi$ & 1.300 & 0.151 & -0.668 & -0.888 & 1.083 & 0.153 & -0.671 & -1.099 \\
\hline
\end{tabular}

\begin{tabular}{|c|c|c|c|c|c|c|c|c|}
\hline $\begin{array}{ll}k & s_{1,2}^{+}\end{array}$ & \multicolumn{4}{|c|}{0.40} & \multicolumn{4}{|c|}{0.60} \\
\hline 0 & 0.942 & 0.589 & -0.670 & -1.167 & 0.837 & 0.579 & -0.670 & -1.750 \\
\hline$\pi / 4$ & 0.938 & 0.542 & -0.670 & -1.190 & 0.831 & 0.537 & -0.670 & -1.785 \\
\hline$\pi / 2$ & 0.934 & 0.412 & -0.671 & -1.258 & 0.821 & 0.412 & -0.671 & -1.900 \\
\hline $3 / 4 \pi$ & 0.931 & 0.239 & -0.671 & -1.370 & 0.815 & 0.24 .2 & -9.672 & -2.092 \\
\hline$\pi$ & 0.929 & 0.155 & -0.672 & -1.461 & 0.812 & 0.157 & -0.673 & -2.214 \\
\hline
\end{tabular}

\begin{tabular}{l|llll}
\hline \multicolumn{1}{l|}{$S_{1,2}^{+}$} & \multicolumn{5}{|c}{0.80} \\
\hline 0 & 0.763 & 0.564 & -0.670 & -3.500 \\
$\pi / 4$ & 0.752 & 0.528 & -0.670 & -3.593 \\
$\pi / 2$ & 0.736 & 0.410 & -0.671 & -3.920 \\
$3 / 4 \pi$ & 0.725 & 0.244 & -0.672 & -4.418 \\
$\pi$ & 0.722 & 0.160 & -0.673 & -4.745
\end{tabular}

In the first instance, the change in the eigenvalue equation is localized on the side chain of the model polymer. This has a very interesting effect on the different bands. Looking at the matrix for the isolated $\mathrm{X}-\mathrm{Y}$ unit, we can identify two eigenvalues $\left(0.47 \beta\right.$ and $-0.67 \beta$ when $\left.S_{3,4}=0.70\right)$ which correspond to the $\mathrm{X}-\mathrm{Y}$ bond; it appears that the first (lowest) band and the third band of Fig. 2 correspond to these eigenvalues. As concerns the third band, it is so narrow that it can be considered to be merely a highly degenerated level identical with the higher level of the $\mathrm{X}-\mathrm{Y}$ bond. The first band, on the other hand, is somewhat wider, and much lower than the lower level of the $X-Y$ bond. The very fact that it is so narrow strongly points to the correspondence just mentioned. 
The two other bands, which should correspond to the two equal nonbonding levels of the isolated unit, are very broad; this is not surprising because the matrices $\mathrm{S}^{+}$and $\mathbf{H}^{+}$contain large elements connecting the orbitals of adjacent $\mathrm{X}$ atoms. An increase in the $\mathrm{X}-\mathrm{Y}$ overlap integral has the effect expected from Eq. (4) on the third band. The effect on the other bands follows to some extent the same trend, except for the upper end of the fourth band. All this is in accordance with the assignment just given for the bands. What may seem more surprising is that the increase of $S_{3,4}$ makes all the bands narrower. A probable explanation for this is that by increasing $S_{3,4}$ one actually decreases the relative importance of the inter-unit interactions; of course, this is a relatively minor effect, but it is quite significant for the higher bands.

As regards the shapes of the bands - a very important feature for the calculation of electron mobility - the effect of the introduction of overlap between the monomers is mainly one of increasing the gradient of the energy with respect to the wave number in the upper bands, and decreasing it in the lower bands, due to the corresponding change in width. On the other hand, an increase in the intra-unit overlap has mainly the opposite effect.

We consider now the effect of the change of inter-unit overlap integrals (Tables II, III, Fig. 3). As has been mentioned, there are two types of such

\section{Table III}

The effect of the change of the smaller intercell overlap integrals on the band structure of the investigated model system

\begin{tabular}{|c|c|c|c|c|c|c|c|c|}
\hline$s_{\mu, \nu}^{+}$ & \multicolumn{4}{|c|}{0} & \multicolumn{4}{|c|}{0.05} \\
\hline 0 & 0.943 & 0.590 & -0.670 & -2.333 & 0.852 & 0.587 & -0.670 & -2.333 \\
\hline$\pi / 4$ & 0.900 & 0.560 & -0.670 & -2.340 & 0.826 & 0.545 & -0.670 & -2.359 \\
\hline$\pi / 2$ & 0.807 & 0.405 & -0.671 & -2.365 & 0.786 & 0.407 & -0.671 & -2.450 \\
\hline $3 / 4 \pi$ & 0.773 & 0.210 & -0.672 & -2.390 & 0.770 & 0.229 & -0.672 & -2.540 \\
\hline$\pi$ & 0.765 & 0.136 & -0.673 & -2.405 & 0.765 & 0.146 & -0.673 & -2.595 \\
\hline$k$ & \multicolumn{4}{|c|}{0.10} & \multicolumn{4}{|c|}{0.15} \\
\hline 0 & 0.796 & 0.572 & -0.670 & -2.333 & 0.771 & 0.547 & -0.670 & -2.333 \\
\hline$\pi / 4$ & 0.788 & 0.533 & -0.670 & -2.385 & 0.784 & 0.504 & -0.671 & -2.584 \\
\hline$\pi / 2$ & 0.775 & 0.410 & -0.671 & -2.553 & 0.779 & 0.400 & -0.671 & -2.860 \\
\hline $3 / 4 \pi$ & 0.767 & 0.243 & -0.672 & -2.834 & 0.773 & 0.256 & -0.672 & -3.292 \\
\hline$\pi$ & 0.765 & 0.159 & -0.673 & -3.010 & 0.765 & 0.174 & -0.673 & -4.001 \\
\hline
\end{tabular}


integrals: the main $X-X$ overlap integral $\left(S_{1,2}^{+}\right)$and the (smaller) overlap integrals not corresponding to chemical bonds.

The introduction of $S_{1,2}^{+}$has the effect already indicated; its increase has a most dramatic effect on the width and positions of the highest unfilled band,

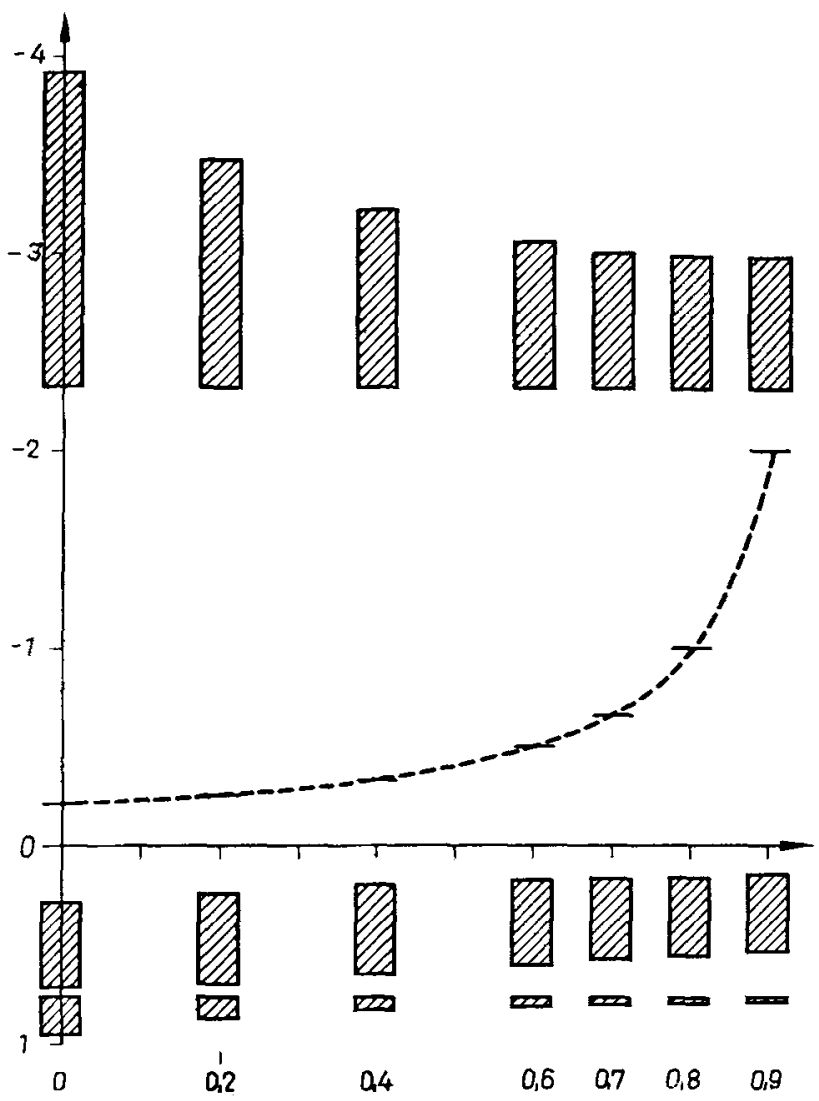

Fig. 2. The change of the band structure of the model system under consideration in the first series of calculations

while the other bands, and in particular the third one, do not show very important changes. If our calculation described a real system, one could conclude that too low an estimate of the $X-X$ overlap integral would not seriously affect a possible hole conduction; on the other hand it might lead to very dubious result as regards electronic conduction due to the opposing effect of the upper band widths and the energy gaps.

The small overlap integrals not associated with chemical bonds could be expected to have a minor effect; surprisingly enough, this is not at all the case, especially for the upper band, but also for the valence band. Note, in particular, 
that a change of the integrals in question from 0.05 to 0.10 already gives a change of more than $100 \%$ in the width of the upper band, and a $10 \%$ change in the width of the valence band. This conclusion speaks for itself; we just mention that in the case of DNA [7], where we have a large number of small

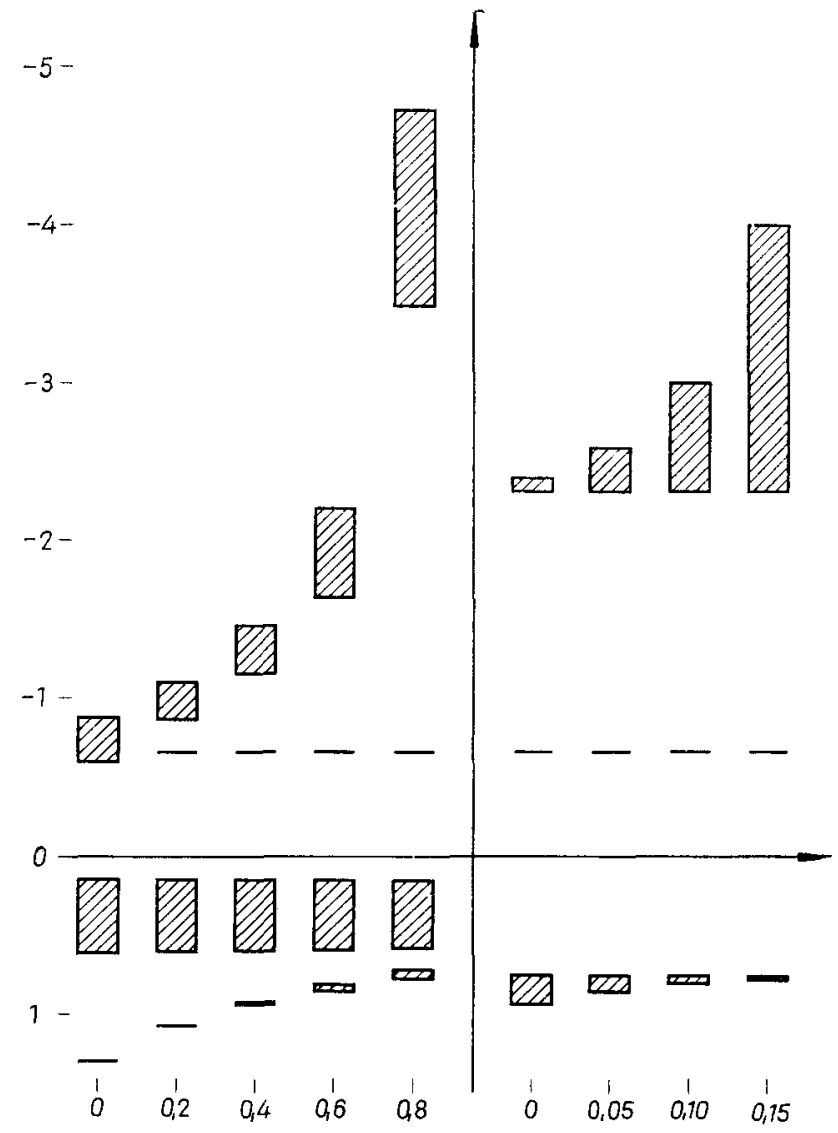

Fig. 3. The change of the band structure of the model system under consideration in the second and third series of calculations

overlap integrals between the superimposed bases, their inclusion in the band calculations might be quite important.

\section{Acknowledgment}

We should like to express our gratitude to Academician G. SCHAy for his encouraging interest and for the warm hospitality given to one of us (G. D. R.). We also wish to acknowledge the scientific and financial support of the Italian National Council of Research which greatly facilitated our collaboration. We are further indebted to Dr. G. Brczó for advice and discussions. 


\title{
REFERENCES
}

1. J. Ladik, Acta Phys. Hung., 18, 173, 1965.

2. J. LadiK, Acta Phys. Hung., 18, 185, 1965.

3. G. Berthier, G. Del Re and A. Veillard, Nuovo Cimento, 44, 3151966.

4. G. Des Re, Nuovo Cimento, 17, 644, 1960.

5. P.-O. Löwdin, J. Chem. Phys., 18, 365, 1950.

6. G. Del Re, J. LadiK and G. Biczó, Phys. Rev., 155, 997, 1967.

7. J. LAdiK and K. Appex, J. Chem. Phys., 40, 2470, 1964.

J. LADik and G. Biczó, J. Chem. Phys., 42, 1658, 1965.

8. G. DeL Re, in Electronic Aspects of Biochemistry, ed. B. Pullman, Academic Press, 1964, p. 221 .

\section{ОБ ЭФФЕКТЕ ВКЛЮЧЕНИЯ ПЕРЕКРЫТИЯ В ВЫЧИСЛЕНИЯ ТЕСНО-СВЯЗАННЫХ ЗОН ТВЕРДЫХ ТЕЛ}

\author{
Г. ДЕЛ РЭ, И. ЛАДИК И М. КАРПЕНТИЭРИ
}

Р езюме

С целью исследования эффекта вклюмения интегралов перекрытия в приближение тесной связи определяется структура энергетических зон модельного полимера, содержащего два центра и четыре орбиты в элементарной ячейке. В первой серии вычислений систематически было изменимо значение интеграла перекрытия внутр ячеек, во второй и третьей сериях - значение большого и небольшого интеграла перекрытия между ячейками соответственно. По отношению полученных результатов изменения интегралов перекрытия как внутри ячеек, так и между ячейками (также и в случае небольших интегралов перекрытия между ячейками) вызвали довольно большие изменения в структуре зон. Отсюда получается вывод, что обычное пренебрежение интегралами перекрытия в приближения тесной связи структуры полос полимеров не обосновано. 